Int. J. Electrochem. Sci., 14 (2019) 5950 - 5960

International Journal of

ELECTROCHEMICAL

SCIENCE

www.electrochemsci.org

\title{
Facile Synthesis of three-dimensional Carbon Nanocages with Hierarchical Porous Structures as Supercapacitor Electrode Materials
}

Liang Jiang, ${ }^{1, *}$, Jing Wang, ${ }^{2, *}$ Yujie Li $^{1}$, Qianqian Zhai ${ }^{2}$, Xuyan Mao ${ }^{1}$, Xiangyu Xu ${ }^{1}$, Jie Yang ${ }^{1}$, Shifeng Hou ${ }^{l}$

${ }^{1}$ Bio-Nano \& Medical Engineering Institute, Jining Medical University, 133 Hehua Road, Jining, 272067, China.

${ }^{2}$ Physics and Information Engineering Department, Jining University, 1 Xingtan Road, Qufu, 273155, China.

*E-mail: jiangliangjnmc@ 126.com (Liang Jiang), wangjingouc@ 126.com (Jing Wang).

doi: $10.20964 / 2019.07 .62$

Received: 2 March 2019 / Accepted: 5 May 2019 / Published: 10 June 2019

Hierarchical carbon with a three-dimensional (3D) structure has become a promising electrode material for supercapacitors due to its excellent rate capability. High surface area hierarchical structure carbon nanocages (HHNCs) were prepared using magnesium oxide as a template with in situ chemical activation. These materials feature a 3D network structure interconnected by carbon nanocages, a high specific surface area $\left(2073 \mathrm{~m}^{2} \mathrm{~g}^{-1}\right)$, a high pore volume $\left(3.17 \mathrm{~cm}^{3} \mathrm{~g}^{-1}\right)$ and a multiscale pore size distribution. Because of the synergistic effects of these advantages, as supercapacitor electrode materials, HHNCs show excellent electrochemical performance with a high specific capacitance $\left(276 \mathrm{~F} \mathrm{~g}^{-1}\right.$ at 0.05 $\mathrm{A} \mathrm{g}^{-1}$ ) and an excellent rate capability (205 $\mathrm{F} \mathrm{g} \mathrm{g}^{-1}$ at $\left.20 \mathrm{~A} \mathrm{~g}^{-1}\right)$. This makes HHNCs promising supercapacitor electrode materials with many potential applications.

Keywords: Supercapacitor; Carbon nanocages; Hierarchical structure; Hard template; In situ chemical activation.

\section{$\underline{\text { FULL TEXT }}$}

(C) 2019 The Authors. Published by ESG (www.electrochemsci.org). This article is an open access article distributed under the terms and conditions of the Creative Commons Attribution license (http://creativecommons.org/licenses/by/4.0/). 